\title{
Cerebral Toxoplasmosis in a Patient with Chronic Lymphocytic Leukemia Treated With Obinutuzumab- Chlorambucil
}

\author{
Hélène Vellemans $^{1 *}$, Marc André ${ }^{1}$, Paul Delrée ${ }^{2}$, Bérangère Devalet ${ }^{1}$, Julien Depaus ${ }^{1}$, Elodie \\ Collinge ${ }^{1}$ and Anne Sonet ${ }^{1}$ \\ ${ }^{1}$ Department of Hematology, CHU UCL Namur, Belgium
}

${ }^{2}$ Departement of Anatomopathology, Institute of Pathology and Genetics, Belgium

*Corresponding author: Vellemans Hélène, Hematology, University CHU UCL Namur, 1, Avenue du Docteur Gaston Thérasse, 5530 Yvoir, Tel: 0032472385191.

To Cite This Article: Hélène Vellemans, Marc André, Paul Delrée, Bérangère Devalet, Julien Depaus, et al., Cerebral Toxoplasmosis in a Patient with Chronic Lymphocytic Leukemia Treated With Obinutuzumab-Chlorambucil. Am J Biomed Sci \& Res. 2021 - 14(2). AJBSR.MS.ID.001968. DOI: 10.34297/AJBSR.2021.14.001968.

Received: 眥September 07, 2021; Published: 制 September 17, 2021

\begin{abstract}
Despite therapeutic advances, infectious complications continue to play a significant role in the clinical course of patients with CLL. Cerebral toxoplasmosis is among the most common CNS infections in immunocompromised patients. Toxoplasma gondii can cause a life threatening CNS or disseminated infection, usually due to reactivation of a latent infection. We describe here the case of a 76 year-old man who developed toxoplasmic encephalitis after a treatment with obinutuzumab-chlorambucil for a CLL. He was admitted to the emergency room for confusion, agitation, Brocca aphasia, diplopia and left mydriasis seven months after the end of treatment with obinutuzumab-chlorambucil. Brain MRI showed at least eight intra-axial supra-tentorial lesions. A brain biopsy was performed and showed a florid toxoplasmosis. Rapidly, treatment with TMP-SMX was started and an improvement in the patient neurological condition was gradually observed. This is the first case of cerebral toxoplasmosis described after treatment with obinutuzumab-chlorambucil in a patient with CLL.
\end{abstract}

Keywords: Chronic lymphocytic leukemia, Cerebral toxoplasmosis, Obinutuzumab, Chlorambucil

\section{Introduction}

In patients diagnosed with hematologic malignancies, treatment with T-cell suppressing chemotherapy increases the risk of central nervous system (CNS) infections [1].

Toxoplasmosis is one of the most common parasitic infections and is caused by a protozoan, Toxoplasma gondii. It is a wellrecognized opportunistic infection usually due to reactivation of a latent infection. In immunocompromised patients, it can cause a life-threatening CNS or disseminated infection. The frequency of this opportunistic infection in patients treated with antineoplastic chemotherapy in the modern era is unknown, as most series were published in patients with bone marrow transplant, and before the widespread use of trimethoprim-sulfamethoxazole (TMP-SMX) prophylaxis or the advent of immunotherapy, including rituximab
[2]. Toxoplasma gondii is a ubiquitous obligate intracellular protozoan parasite in humans and animals. During the acute stage of the infection, tachyzoites quickly proliferate within a variety of nucleated cells and spread throughout host tissues. Following the acute stage, the parasite forms cysts (latent stage) in various organs, especially the brain, heart, and skeletal muscle, establishing chronic infection.

Bradyzoïtes containing brain cysts may become reactivated, i.e, reconvert into cytotoxic tachyzoites during secondary immune deficiency and cause toxoplasmic encephalitis, in particular in individuals with HIV/AIDS or organ transplants [3]. It can cause focal or disseminated brain lesions leading to neurological deficit, coma and death [4]. 
Here we described the case of an old man who developed toxoplasmic encephalitis after a treatment with obinutuzumabchlorambucil for a chronic lymphocytic leukemia (CLL).

\section{Case Report/Case Presentation}

A 76-year-old man was diagnosed in 2008 with CLL stage Binet A (13q14 deletion). He was regularly monitored in this context. In October 2018, the patient developed auto-immune hemolytic anemia and grade III thrombocytopenia, classifying CLL as stage Binet C. He was first, admitted to our department in December 2018, because of fever and elevation of LDH. An aggressive transformation of the CLL (Richter's syndrome) was excluded by PET-CT. Given the age, obinutuzumab-chlorambucil was started on January 2019. Five of six cycles were administered due to hematological toxicity. The treatment ended in May 2019 with persistent anemia.

Seven months later, the patient presented to the emergency for confusion, agitation, Brocca aphasia, diplopia and left mydriasis. Biology showed essentially lymphocytopenia (160 ly/ $\mu \mathrm{l}$ ). Brain MRI on 13/12/2019 showed at least eight intra-axial supra- tentorial lesions. Three of them were supracentimetric with marked perilesional edema predominant in the left parietal (Figure 1), compatible with secondary lesions (no argument for lymphoma or PML). In 2018, toxoplasma serology was IgG positive. An exhaustive bacteriological assessment was carried out with, among others, search for cryptococcus, herpes simplex and toxoplasma gondii in CSF: negative. Thereby, a brain biopsy was performed. It showed a florid toxoplasmosis, associated with a major chronic inflammatory reaction (encephalitis) and with a range of supracentimetric ischemic necrosis probably secondary to significant chronic hypertrophic meningitis with vascular lesions notably comprising partially recanalized thromboses. Inflammation begins on the wall of meningeal vessels (vasculitis or more probably inflammation secondary to the phenomena of thrombosis). The results highlight cerebral toxoplasmosis (Figure 2). Treatment with high dose TMP/SMX was started. After six days of treatment, TMP/ SMX was stopped because of major hyponatremia and shifted to clindamycin $600 \mathrm{mg}$ four times a day and pyrimethamine $75 \mathrm{mg}$ once daily for a total duration of 42 days. An improvement in the patient's neurological condition was gradually observed.

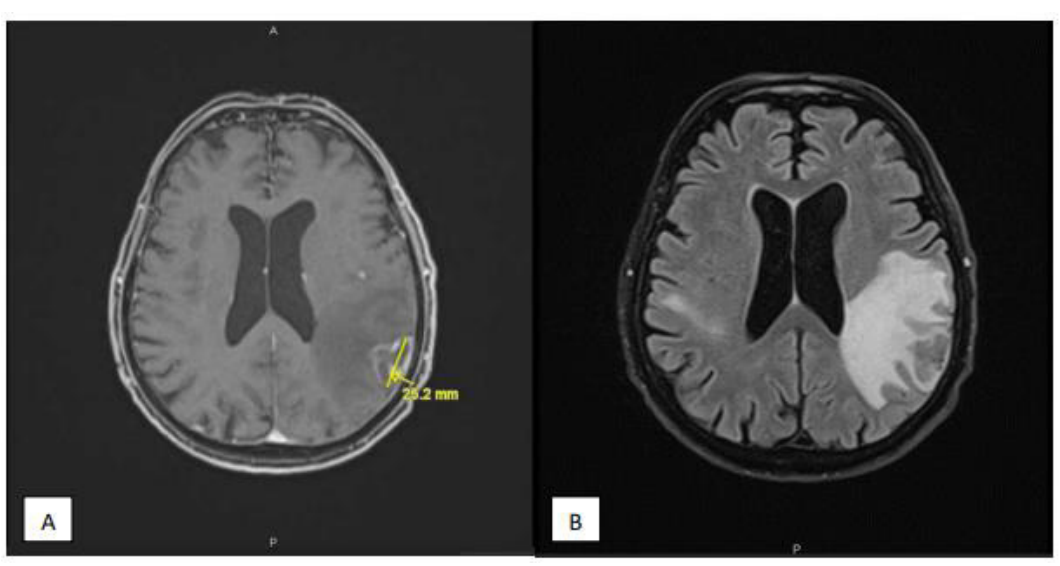

Figure 1: MRI shows supracentimetric supra-tentorial toxoplasma lesion (A) and marked perilesional edema surrounding that lesion (B).

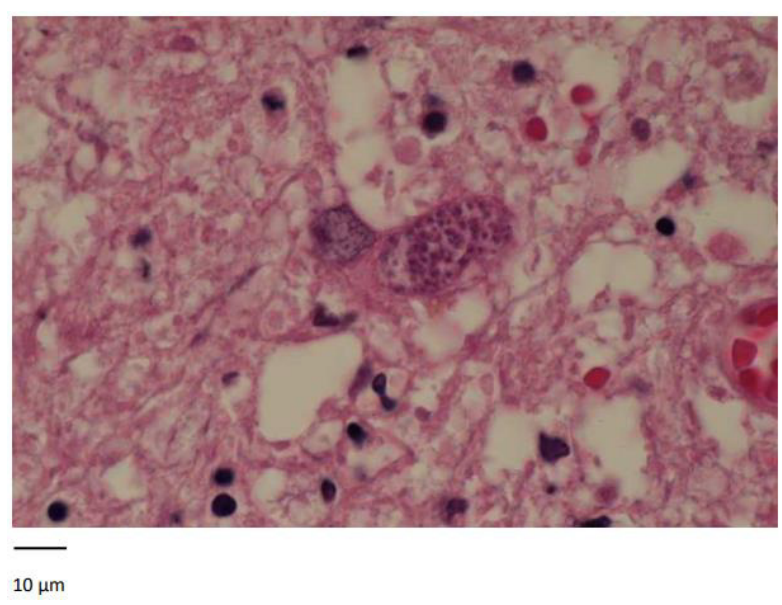

Figure 2: Toxoplasmic pseudo cyst consisting of multiplied protozoa within a cell, HE staining. 


\section{Discussion}

Our case illustrates the first example of cerebral toxoplasmosis in a patient with CLL treated with obinutuzumab-chlorambucil.

In patients with hematological malignancies, especially those after previous allogeneic bone marrow or stem cell transplantation, the incidence of various CNS infections may be up to $15 \%$. Among causative organisms, toxoplasma gondii is the most prevalent [5]. Few cases of cerebral toxoplasmosis have been reported in patients with CLL, and to our knowledge, no cases after treatment with obinutuzumab-chlorambucil. The most common cause of death in patients with CLL is infections due to progressive immunodeficiency. The pathogenesis of these complications is twofold, with immune defects associated with the primary disease and compounded by chemotherapy [6].

One of the most profound hematological effects of the chemotherapy is severe lymphopenia, often predisposing to a variety of infectious complications such as Herpes simplex virus, cytomegalovirus and Pneumocystis jiroveci pneumonia. Infections may be related to the quantitative and qualitative T-cell abnormalities induced by chemotherapeutic agents. The impact is greater on CD4+ and may persist for 1 to 2 years after discontinuation of therapy.

Obinutuzumab (Gazyvaro ${ }^{\circledR}$ ) is a type II humanised anti-CD20 recombinant monoclonal antibody. Obinutuzumab in combination with chlorambucil (Leukeran ${ }^{\circledR}$ ) is indicated for the treatment of previously untreated CLL in elderly unfit patients. Due to the mode of action, a depletion of B lymphocytes caused by the anti CD20 antibody is to be anticipated during treatment [7].

Despite therapeutic advances, infectious complications continue to play a significant role in the clinical course of patients with CLL. Historically, cerebral toxoplasmosis was among the most common CNS infections in untreated immunocompromised HIV patients [8]. A recent retrospective multicenter French study of 180 PCR-positive toxoplasmosis cases in immunocompromised patients found that $14 \%$ of cases occurred in the non-transplant, non-HIV-infected group, especially in patients with hematologic malignancy and connective tissue disease on immunosuppressive medications [9].

Brain imaging with computed tomography or magnetic resonance is essential for the diagnosis of toxoplasma lesions. However, MRI should be used as the initial choice if there is a high clinical suspicion, due to its greater sensitivity than CT [10].

Definitive diagnosis of infection by Toxoplasma Gondii requires identification of tachyzoites in biopsy samples. A brain biopsy provides a definitive diagnosis of toxoplasmosis infection in most cases.
The cornerstone of treatment is a combination of pyrimethamine or trimethoprim-sulfamethoxazole, sulfadiazine or clindamycin. Actually, evidence fails to identify a superior regimen in terms of relative efficacy or safety for the treatment of cerebral toxoplasmosis. Real-world considerations are relevant when TMP-SMX is evaluated as a preferred treatment for cerebral toxoplasmosis. Potential advantages of TMP-SMX include the convenience of the lower pill burden and dosing frequency and the availability of intravenous formulations. The availability of several generic has an impact on cost-effectiveness and increased accessibility. Current guidelines suggest the option of intravenous TMP-SMX as initial treatment in severely ill patients [11].

\section{Conclusion}

With the advent of new monoclonal antibodies and the associated lymphocytopenia, there is an evolving need of recommendation for prevention and management of opportunistic infections in patients receiving these agents. It is essential to think of opportunistic cerebral infections even at distance from therapy. Indeed, early suspicion and prompt treatment during the initial phase of cerebral toxoplasmosis reduce the risk of neurological sequelae and death. Brain MRI and prompt brain biopsy can provide rapid diagnosis and adequate treatment of cerebral toxoplasmosis.

\section{Acknowledgement}

I thank Prof. A Sonet for the proofreading, my colleagues for allowing me to write this case report and Doctor Delrée, anatomopathologist, who analyzed the brain biopsy.

\section{Statement of Ethics}

We ensure the accuracy, quality, and integrity of this case report. No identifying patient information was disclosed. The ethics committee of the CHU UCL Namur approved the drafting of the case report.

\section{Conflict Interest}

The authors have no conflicts of interest to declare.

\section{Author Contributions}

Hélène Vellemans: literature review and manuscript writing. Anne Sonet: Proofreading. Anne Sonet, Julien Depaus, Bérangère Devalet, Elodie Collinge: clinical follow-up. Paul Delrée: analysis of the biopsy samples.

\section{Data Availability Statement}

All data generated or analyzed during this case report are included in this article and its supplementary material files. Further enquiries can be directed to the corresponding author. 


\section{References}

1. Xu J, Rod J Nault, Andres Maldonado Naranjo, Leonardo A Frizon, Kuruvilla John, et al. (2018) Disseminated cerebral toxoplasmosis in a patient with chronic lymphocytic leukemia. J Clin Neurosci 50: 127-128.

2. Chintakuntlawar A, Michael Kidd, Aref Al Kali, Walter Wilson, Carrie A ThompsonT (2015) oxoplasmosis in patients with hematologic malignancies. Leuk Lymphoma 56(2): 536-538.

3. Schluter D, Barragan A (2019) Advances and challenges in Understanding cerebral Toxoplasmosis. Front Immunol 10: 242.

4. Salman Abbasi Far, Ali Khajeh, Alireza Khosravi, Afsaneh Mirshekar Safoora Masoumi, et al. (2020) Fulminant and diffuse cerebral toxoplasmosis as the first manifestation of HIV infection: A case presentation and review of the literature. Am J Case Rep 21: e919624.

5. Savsek L, Opaskar T (2016) Cerebral toxoplasmosis in a diffuse large B cell lymphoma patient. Radiol Oncol 50(1): 87-93.

6. Abedalthagafi M, Elisabeth J Rushing, David Garvin, Bruce Cheson, Metin Ozdemirli, et al. (2010) Asymptomatic diffuse encephalitic cerebral toxoplasmosis in patient with chronic lymhocytic leukemia: case report and review of the literature. Int J Clin Exp Pathol 3(1): 106-109.

7. (2021) Product monograph, GAZYVA.

8. San Andrés FJ, Rafael Rubio, Jesús Castilla, Federico Pulido, Guillermo Palao, et al. (2003) Incidence of acquired immunodeficiency syndromeassociated opportunistic diseases and the effect of treatment cohort of 1115 patients infected with human immunodeficiency virus, 19891997. Clin Infect Dis 36(9): 1177-1185.

9. Dunay IR, Gajurel K, Dhakal R, Liesenfeld O, Montoya JG (2018) Treatment of toxoplasmosis: historical perspective, animal models, and current clinical practice. Clin Microbiol Rev 31(4): e00057-17.

10. Levy RM, Rosenbloom S, Perrett LV (1986) Neuroradiologic findings in AIDS: A review of 200 cases. Am J Roentgenol 147(5): 977-983.

11. José Ernesto Vidal (2019) HIV-related cerebral toxoplasmosis revisited: current controversies of an old disease. J Int Assoc Provid AIDS care 18: $1-20$ 